\title{
Case Report Leclercia adecarboxylata in an immunocompetent
patient
}

\author{
Benjamin Hess, ${ }^{1}$ Andrew Burchett ${ }^{2}$ and Mark K. Huntington ${ }^{1}$ \\ ${ }^{1}$ Center for Family Medicine, 1115 East Twentieth Street, Sioux Falls, SD 57105, USA \\ ${ }^{2}$ McGreevy Clinic, 1200 South Seventh Avenue, Sioux Falls, SD 57105, USA
}

Correspondence

Mark K. Huntington

mark.huntington@usd.edu

\begin{abstract}
Leclercia adecarboxylata is a rarely reported human pathogen, most commonly affecting immunocompromised individuals. In reported cases of immunocompetent patients infected with this organism, it is seen exclusively in the context of polymicrobial infections. We report here the case of an abscess in an immunocompetent patient that grew out $L$. adecarboxylata as a pure culture. The limited literature available on this organism is reviewed, and the potential implication of this finding is discussed.
\end{abstract}

Received 2 October 2007

\section{Introduction}

Leclercia adecarboxylata is a motile, Gram-negative rod, formerly identified as Escherichia adecarboxylata (Armentrout \& Brown, 1981; Daza et al., 1993; Izard et al., 1985; Leclerc, 1962; Pokhil, 1996; Rice et al., 1991; Richard, 1989; Spierings et al., 1993; Tamura et al., 1986). Although generally sensitive to most antibiotics (Stock et al., 2004; Temesgen et al., 1997), there is a report of resistant strains (Mazzariol et al., 2003).

Since its initial identification in 1962 (Leclerc, 1962), only a score of clinical reports have been published; the majority of affected patients described were immunocompromised in some way (Beltran et al., 2004; Cai et al., 1992; Daza et al., 1993; de Baere et al., 2001; Dudkiewicz \& Szewczyk, 1993; Fattal \& Deville, 2000; Lee et al., 1999; Longhurst \& West, 2001; Martinez et al., 1998; Mazzariol et al., 2003; Otani \& Bruckner, 1991; Perez-Moreno et al., 2003; Rodriguez et al., 2001; Sawamura et al., 2005; Temesgen et al., 1997). In those described as previously healthy, the organism was present as part of a post-traumatic polymicrobial infection (Greco et al., 2001; Temesgen et al., 1997). We report here an infection by L. adecarboxylata as the sole pathogen causing infection in an immunocompetent woman.

\section{Case report}

A 40-year-old previously healthy female presented to an urgent care clinic with a wound on her right foot. Four weeks previously, she had received a pedicure during which the technician inadvertently nicked her heel with an instrument, leaving a very small, superficial incision. Later, she went swimming in a chlorinated public swimming pool. Over the interval from injury to presentation, a slow-growing abscess developed and subsequently began draining. The patient was a runner, primarily utilizing asphalt surfaces, and the lesion caused her significant discomfort, which prompted the visit to the clinic. Her past medical history was significant only for remote thyroiditis, mitral valve prolapse, a mood disorder and mild pedal oedema. Her current medications were limited to bupropion and hydrochlorothiazide.

Examination revealed she was afebrile, non-toxic, and not in distress. On her heel was a $2 \mathrm{~cm}$ bulla with a stellate break in the overlying callous. Localized erythema was present, without significant spread beyond the raised area. The area was slightly tender to palpation. An X-ray showed no perioesteal reaction. A small quantity of clear, nonpurulent drainage was expressed and cultured. The patient was placed on moxifloxacin empirically; ultimately the culture was reported as showing heavy growth of Leclercia adecarboxylata, which was identified by its biochemical profile and antibiotic susceptibility (Stock et al., 2004) utilizing a Vitek 2 automated microbiological system. No other organisms, aerobic or anaerobic, were present. Because of this organism's strong association with being immunocompromised, the patient was recalled for an evaluation of comorbidities that could result in a depressed immune status. None was identified. Following the course of antibiotics, the infection resolved without complication or sequela.

\section{Discussion}

L. adecarboxylata is a member of the Enterobacteriaceae family, the members of which are regarded as normal flora in the gut of animals, and has been isolated from human stool (Cai et al., 1992). It has also been isolated from the skin of an asymptomatic blood donor (Davenport \& Land, 2007) and from a variety of environmental sources, including one strain from an oil-spill site that was capable of utilizing polyaromatic hydrocarbons as its sole carbon source (Sarma et al., 2004). The organism is cosmopolitan, 
with cases reported from around the globe (Cai et al., 1992; Daza et al., 1993; Dudkiewicz \& Szewczyk, 1993; Greco et al., 2001; Leclerc, 1962; Lee et al., 1999; Martinez et al., 1998; Sawamura et al., 2005; Temesgen et al., 1997).

The epidemiological significance of $L$. adecarboxylata is not clear. The paucity of reports of human infection may reflect misdiagnosis, as the organism shares many biochemical features with E. coli, rather than a true infrequency of human infection. Increased routine use of antimicrobial susceptibility patterns (Stock et al., 2004) and molecular typing (Woo et al., 2001) in the clinical laboratory will likely reveal a more complete picture of the extent of this organism's effects on human health.

In at least 12 of the 18 published cases of infection involving this organism, clear evidence of being immunocompromised was present in the affected patients (Table 1). This led to the suggestion by some authors that it is exclusively an opportunistic pathogen in humans (Mazzariol et al., 2003; Stock et al., 2004). An unusual feature of this organism is its frequent association with polymicrobial infections (de Baere et al., 2001; Lee et al.,
1999; Longhurst \& West, 2001; Rodriguez et al., 2001; Sawamura et al., 2005; Temesgen et al., 1997), particularly in previously healthy, immunocompetent patients.

Referring to Table 1, the association between a lack of any known immunodeficiency and the presence of polymicrobial cultures is striking. It suggests that $L$. adecarboxylata is dependent on the presence of other pathogens in order to cause disease. This raises the possibility that some synergism exists between the organisms, enhancing the virulence of $L$. adecarboxylata. Further study is needed to elucidate whether this is the case, and if so, whether is it due to modification of the microenvironment or the transfer of genetic factors via the well-known promiscuity of Gram-negative bacteria.

The infection reported in this case is of interest in that it is believed to be the first report in the literature of a pure culture - without other coinciding pathogens - of $L$. adecarboxylata from a wound infection of an immunocompetent patient. The success of this strain in infecting a healthy individual without the apparent aid of other organisms suggests that it may possess unique virulence

Table 1. Association between immune status and polymicrobial cultures in $L$. adecarboxylata infections

\begin{tabular}{|c|c|c|c|}
\hline Immunodeficiency & Culture source & Bacterial/fungal co-infection & Reference \\
\hline None & Wound & $\begin{array}{l}\text { Enterobacter cloacae, Citrobacter freundii, Klebsiella } \\
\text { pneumoniae, Stenotrophomonas maltophilia, } \\
\text { Corynebacterium, Acremonium, Penicillium, Mucor, } \\
\text { Enterococcus, Geotrichum }\end{array}$ & Temesgen et al. (1997) \\
\hline None & Wound & Acinetobecter calcoaceticus, Enterobacter agglomerans & Temesgen et al. (1997) \\
\hline None & Wound & Staphylococcus epidermidis & Greco et al. (2001) \\
\hline None & Wound & $\begin{array}{l}\text { Shewanella putrefaciens, Enterobacter cloacae, Group B } \\
\text { streptococcus }\end{array}$ & Greco et al. (2001) \\
\hline $\begin{array}{l}\text { Infant with intestinal } \\
\text { atresia, on parenteral } \\
\text { nutrition }\end{array}$ & Blood & None & Otani \& Bruckner (1991) \\
\hline Chemotherapy & Blood & Escherichia hermannii & Lee et al. (1999) \\
\hline Dialysis & Peritoneal fluid & None & Fattal \& Deville (2000) \\
\hline Dialysis & Peritoneal fluid & Acinetobacter lwoffii & Rodriguez et al. (2001) \\
\hline Renal transplant & Urine & None & Woo et al. (2001) \\
\hline Renal transplant & Blood & None & Greco et al. (2001) \\
\hline Candida sepsis & Gall bladder & None & de Baere et al. (2001) \\
\hline Candida sepsis & Blood & None & de Baere et al. (2001) \\
\hline Leukaemia & Blood & Staphylococcus aureus & Longhurst \& West (2001) \\
\hline Leukaemia & Blood & None & Mazzariol et al. (2003) \\
\hline $\begin{array}{l}\text { Unknown (undiagnosed } \\
\text { chronic diarrhoea) }\end{array}$ & Synovial fluid & None & Perez-Moreno et al. (2003) \\
\hline
\end{tabular}


factors that are absent from those strains responsible for the earlier reported cases. The day the culture results were reported, efforts were made to acquire a specimen for further biochemical and molecular analysis. Tragically, the culture had already been discarded by the clinical laboratory, per their routine practice for Enterobacteriaceae isolates.

\section{References}

Armentrout, R. W. \& Brown, R. D. (1981). Molecular cloning of genes for cellobiose utilization and their expression in Escherichia coli. Appl Environ Microbiol 41, 1355-1362.

Beltran, A., Molinero, A. V., Capilla, S. \& Polo, A. M. (2004). Isolation of Leclercia adecarboxylata from wound exudate of a diabetic patient. Med Clin (Barc) 122, 159.

Cai, M., Dong, X., Wei, J., Yang, F., Xu, D., Zhang, H., Zheng, X., Wang, S. \& Jin, H. (1992). Isolation and identification of Leclercia adecarboxylate in clinical isolates in China. Wei Sheng Wu Xue Bao 32, 119-123.

Davenport, P. \& Land, K. J. (2007). Isolation of Leclercia adecarboxylata from the blood culture of an asymptomatic platelet donor. Transfusion 47, 1816-1819.

Daza, R. M., Iborra, J., Alonso, N., Vera, I., Portero, F. \& Mendaza, P. (1993). Isolation of Leclercia adecarboxylata in a cirrhotic patient. Enferm Infecc Microbiol Clin 11, 53-54.

de Baere, T., Wauters, G., Huylenbroeck, A., Claeys, G., Peleman, R., Verschraegen, G., Allemeersch, D. \& Vaneechoutte, M. (2001). Isolations of Leclercia adecarboxylata from a patient with a chronically inflamed gallbladder and from a patient with sepsis without focus. J Clin Microbiol 39, 1674-1675.

Dudkiewicz, B. \& Szewczyk, E. (1993). Etiology of bacterial endocarditis in materials from cardiology and cardiac surgery clinics of the Lodz Academy. Med Dosw Mikrobiol 45, 357-359.

Fattal, O. \& Deville, J. G. (2000). Leclercia adecarboxylata peritonitis in a child receiving chronic peritoneal dialysis. Pediatr Nephrol 15, 186-187.

Greco, G., Ostojich, N., Flores, Y., Sanchez, M., Clara, L. \& Ascione, A. (2001). Leclercia adecarboxylata, a new enterobacteriaceae in antique infections. In 39th Annual Meeting of the Infectious Diseases Society of America, San Francisco, CA.

Izard, D., Mergaert, J., Gavini, F., Beji, A., Kersters, K., De Ley, J. \& Leclerc, H. (1985). Separation of Escherichia adecarboxylata from the "Erwinia herbicola-Enterobacter agglomerans" complex and from the other Enterobacteriaceae by nucleic acid and protein electrophoretic techniques. Ann Inst Pasteur Microbiol 136B, 151-168.

Leclerc, H. (1962). Etude biochimique d'enterobacteriaceae pigmentees. Ann Inst Pasteur (Paris) 102, 726-741.

Lee, N. Y., Ki, C. S., Kang, W. K., Peck, K. R., Kim, S. \& Song, J. H. (1999). Hickman catheter-associated bacteremia by Leclercia adecarboxylata and Escherichia hermannii: a case report. Korean J Infect Dis 31, 167-170.
Longhurst, C. A. \& West, D. C. (2001). Isolation of Leclercia adecarboxylata from an infant with acute lymphoblastic leukemia. Clin Infect Dis 32, 1659.

Martinez, M. M., Sanchez, G., Gomez, J., Mendaza, P. \& Daza, R. M. (1998). Isolation of Leclercia adecarboxylata in ulcer exudate. Enferm Infecc Microbiol Clin 16, 345.

Mazzariol, A., Zuliani, J., Fontana, R. \& Cornaglia, G. (2003). Isolation from blood culture of a Leclercia adecarboxylata strain producing an SHV-12 extended-spectrum beta-lactamase. J Clin Microbiol 41, $1738-1739$.

Otani, E. \& Bruckner, D. (1991). Leclercia adecarboxylata isolated from blood culture. Clin Microbiol Newsl 13, 157-158.

Perez-Moreno, M. O., Anguera, J., Carulla, M. \& Perez-Moreno, M. (2003). Septic arthritis due to Leclercia adecarboxylata of probable iatrogenic origin. Enferm Infecc Microbiol Clin 21, 167-168.

Pokhil, S. I. (1996). Species of enterobacteria new to medicine. Mikrobiol Z 58, 94-103.

Rice, E. W., Allen, M. J., Brenner, D. J. \& Edberg, S. C. (1991). Assay for $\beta$-glucuronidase in species of the genus Escherichia and its applications for drinking-water analysis. Appl Environ Microbiol 57, 592-593.

Richard, C. (1989). New Enterobacteriaceae found in medical bacteriology Moellerella wisconsensis, Koserella trabulsii, Leclercia adecarboxylata, Escherichia fergusonii, Enterobacter asbutiae, Rahnella aquatilis. Ann Biol Clin (Paris) 47, 231-236.

Rodriguez, J. A., Sanchez, F. J., Gutierrez, N., Garcia, J. E. \& GarciaRodriguez, J. A. (2001). Bacterial peritonitis due to Leclercia adecarboxylata in a patient undergoing peritoneal dialysis. Enferm Infecc Microbiol Clin 19, 237-238.

Sarma, P. M., Bhattacharya, D., Krishnan, S. \& Lal, B. (2004). Degradation of polycyclic aromatic hydrocarbons by a newly discovered enteric bacterium, Leclercia adecarboxylata. Appl Environ Microbiol 70, 3163-3166.

Sawamura, H., Kawamura, Y., Yasuda, M., Ohkusu, K., Takahashi, Y., Ishihara, S., Deguchi, T. \& Ezaki, T. (2005). A clinical isolate of Leclercia adecarboxylata from a patient of pyelonephritis. Kansenshogaku Zasshi 79, 831-835.

Spierings, G., Ockhuijsen, C., Hofstra, H. \& Tommassen, J. (1993). Polymerase chain reaction for the specific detection of Escherichia coli/Shigella. Res Microbiol 144, 557-564.

Stock, I., Burak, S. \& Wiedemann, B. (2004). Natural antimicrobial susceptibility patterns and biochemical profiles of Leclercia adecarboxylata strains. Clin Microbiol Infect 10, 724-733.

Tamura, K., Sakazaki, R., Kosako, Y. \& Yoshizaki, E. (1986). Leclercia adecarboxylata gen. nov., comb. nov., formerly known as Escherichia adecarboxylata. Curr Microbiol 13, 179-184.

Temesgen, Z., Toal, D. R. \& Cockerill, F. R., III (1997). Leclercia adecarboxylata infections: case report and review. Clin Infect Dis 25, 79-81.

Woo, P. C., Cheung, E. Y., Leung, K. \& Yuen, K. (2001). Identification by $16 \mathrm{~S}$ ribosomal RNA gene sequencing of an Enterobacteriaceae species with ambiguous biochemical profile from a renal transplant recipient. Diagn Microbiol Infect Dis 39, 85-93. 